$P_{\text {resentación }}$

\title{
Contribuciones al dosier
}

Entre los estudios de ciencia, tecnología y sociedad (CTS), filosofía de la ciencia y filosofía de la tecnología abundan posibilidades de interacción, pero, lamentablemente, debido a los prejuicios y los distanciamientos sostenidos desde hace varias décadas, las relaciones entre estos campos siguen siendo muy limitadas. Frente ello, desde el año 2014, los editores formulamos la iniciativa de preparar un número temático para promover los encuentros entre estos campos, que finalmente se materializó en esta publicación. Con el objetivo de convocar la participación de un buen número de autores de diferentes continentes y países, propusimos como estrategia editorial elaborar y realizar en varios idiomas (francés, inglés y español) la publicación de manera conjunta y simultánea entre la revista francesa Revue d'Anthropologie des Connaissances y la revista colombiana Trilogía Ciencia Tecnología Sociedad, esto con el fin de lograr una mayor divulgación.

Los artículos que hacen parte de este número plantean nuevos encuentros entre la filosofía y el campo CTS a través de caminos muy específicos, basados en varias investigaciones en física, química, biología y matemáticas, relacionados con los siguientes temas: la concepción del tiempo-paisaje en la ciencia, la política del ciclo cerrado del combustible en la industria de la energía nuclear, la 
II epistemología de las ciencias de campo, la constitución de la célula viviente como objeto epistémico, y el influjo de algunas dinámicas sociales en el desarrollo de las matemáticas aplicadas. De manera breve, las contribuciones de este dosier desarrollan los siguientes planteamientos:

En el primer artículo, Encuentrosentre filosofía de la ciencia, filosofía dela tecnología CTS se realiza un amplio y detallado estado del arte de las relaciones sostenidas entre los campos mencionados, y se propone que, tras la influencia de las denominadas «guerras de la ciencia», se han sostenido, en general, los distanciamientos entre los campos, aunque en algunos terrenos muy específicos se han modificado los escenarios de interacción, comenzándose a desarrollar encuentros productivos relacionados con algunas concepciones alternativas de la ciencia y la tecnología. Los planteamientos de este texto sirven de marco y antesala a las contribuciones de los demás artículos, donde se muestran ejemplos concretos en disciplinas específicas y de encuentros productivos entre CTS, filosofía de la ciencia y filosofía de la tecnología.

En el siguiente artículo, Fenomenotecnia y sistemas experimentales: el caso de la célula viviente, Juan Carlos Gallego-Gómez y Germán Guerrero-Pino analizan la constitución de la célula viviente como objeto epistémico desde el ensamblaje de muchos procesos experimentales materiales, desarrollados inicialmente en los campos de la teoría celular, la citología y la bioquímica; luego, en la microcinematografía, la microscopía de fluorescencia y la microscopía confocal, hasta llegar al reciente campo de la imagenología de las células vivas. Los autores interpretan el surgimiento de la célula viva como un nuevo espacio de representación inexistente previamente, a partir de los conceptos de fenomenotecnia propuesto por Bachelard, y de sistemas experimentales propuesto por Rheinberger, y muestran cómo la noción de sistemas experimentales logra ampliar y precisar la noción de fenomenotecnia. A través de la articulación de varios aportes de la filosofía de la ciencia y del campo CTS, los autores muestran que los distintos sistemas experimentales involucrados en la constitución de la célula viviente como objeto epistémico fueron arreglos científicos producidos en laboratorios que implicaron la convergencia de aspectos locales, técnicos, instrumentales, institucionales, sociales y epistémicos. Esta compleja articulación de aspectos heterogéneos producida en las prácticas experimentales, no ha sido comprendida adecuadamente desde la concepción tradicional de la ciencia centrada en el estudio de las teorías. 
En el artículo titulado Pensando epistemologías desde el campo, Ezequiel Sosiuk y Emiliano Martín-Valdez estudian la aún poco conocida epistemología de las ciencias de campo. Los autores muestran que el campo es un lugar de producción de conocimientos con condiciones muy específicas, como el laboratorio, donde el análisis de su epistemología puede llegar a ser tan relevante y significativo para los estudios de la ciencia como lo fue el análisis de la epistemología del laboratorio. Los autores examinan detalladamente las condiciones especiales con las que se desarrolla la investigación científica en el campo a la luz de los aportes de algunos estudios recientes sobre las ciencias de campo y de otros aportes de los estudios CTS y de la filosofía de la ciencia. Muestran, además, que algunas de las condiciones más importantes de la investigación en el campo son las siguientes: ella implica contextualizar los objetos de conocimiento, en tanto, el trabajo de campo debe operar sobre un terreno no diseñado para investigar; también implica producir y movilizar conocimientos para controlar y ordenar el lugar de trabajo; y adicionalmente, el trabajo de campo supone adaptar las prácticas experimentales de forma alterna a su desarrollo en el laboratorio. En términos generales, el estudio de las condiciones epistemológicas de las ciencias de campo enriquece la comprensión de las formas como se desarrolla la investigación científica en sus distintos lugares de producción.

Por su parte, en el texto De las eras a los paisajes. Una perspectiva crítica sobre la «era del átomo»: un encuentro entre CTS y filosofía de la tecnología, Bernadette BensaudeVincent argumenta en favor de la adopción de la concepción del tiempo-paisaje -categoría propuesta por Barbara Adam-, o del tiempo desde un punto de vista meteorológico para pensar los cambios tecnológicos y planetarios, en lugar de la afirmación del tiempo cronológico lineal, de la flecha del tiempo, presupuesto en la mayoría de las teorías científicas. A partir del estudio de caso del análisis de las distintas edades del átomo, identificadas en algunos materiales radioactivos producidos por la industria de la energía nuclear, en el texto se cuestiona la afirmación de un tiempo cronológico lineal de avance de las transformaciones de los materiales radioactivos y de las energías hacia el progreso o la catástrofe, y se sustenta la necesidad de adoptar la noción de tiempo-paisaje. A través de una articulación muy elocuente, actual y relevante entre varios campos disciplinares, la autora cuestiona los presupuestos deterministas modernos del tiempo cronológico, y propone una concepción situacional, contextual, interna e inmanente del tiempo, necesaria para replantear la orientación antropocéntrica 
de la narrativa sobre el Antropoceno, y construir una orientación alternativa a partir de la cual se pueda enfrentar la complejidad de los cambios sociales y tecnológicos asociados con esta nueva era geológica. En correspondencia con lo que han planteado varias perspectivas desde el campo CTS y la filosofía de la tecnología, este cambio en la concepción del tiempo supone un giro hacia una ontología plural y plana que reconoce las condiciones ontológicas particulares de las entidades singulares y la simetría entre las capacidades agenciativas o performativas de las cosas, las entidades naturales, las sociedades y las personas.

En el trabajo, Power and Operations. Simondon and the Imaginaries of the Nuclear Industry, Ange Pottin analiza la política del «ciclo cerrado del combustible» asumida por el gobierno francés durante la década de 1970, específicamente las posibilidades imaginadas de regeneración de los residuos radioactivos empleados en los reactores nucleares hasta su máximo uso como combustibles y sus implicaciones sociales. Desde un diálogo fluido entre algunos aportes de la filosofía de la tecnología y del campo CTS, se abordan, en el caso, las críticas a la cultura tecnocrática, formuladas por Gilbert Simondon, y el papel social de las representaciones e imaginarios técnicos planteada fundamentalmente por Sheila Jassanof. A partir de Simondon se muestra el contrasentido entre las posibilidades imaginadas por la mentalidad tecnocrática, desde los intereses de máxima rentabilidad y eficacia del sistema energético, y las posibilidades de las transformaciones de los materiales en las operaciones realizadas por los técnicos. Por su parte, con Jassanof se muestra la función o el papel político que juegan los imaginarios sociotécnicos, especialmente para el sostenimiento y la eficacia de esta política. A través del encuentro entre estas perspectivas se busca también integrar el enfoque normativo de Simondon con el enfoque empírico descriptivo de Jassanof.

Seguidamente, en el texto What is a Theorem (in Practice)? The Role of Metamathematics in the Making of Mathematics, Sylvain Lavau analiza la constitución y consolidación de las comunidades científicas que promovieron el desarrollo de la teoría del control geométrico en la década de 1970 a partir de un estudio bibliométrico sobre las dinámicas de citación y cocitación en las publicaciones científicas realizadas en esta área de estudio de las matemáticas aplicadas. A través del estudio de este caso, el autor muestra cómo las dinámicas sociales de adhesión a una comunidad académica y de discusión al interior de ella, fueron elementos metamatemáticos que influyeron en el desarrollo de esta 
teoría. Específicamente, las discusiones sobre la adopción de métodos geométricos condujeron a un cambio de perspectiva y a la consolidación de la comunidad académica involucrada en el desarrollo de la teoría del control geométrico. En términos generales, con esta investigación el autor justifica la relevancia y la posibilidad de enriquecer el estudio filosófico de las prácticas matemáticas con los aportes de la sociología de las matemáticas y del campo CTS.

Aprovechamos para agradecer a todos los autores e investigadores que participaron de esta convocatoria, pues gracias a ellos se hizo posible la materialización de esta edición temática. Finalmente, teniendo en cuenta el papel esencial de las revistas científicas como medios para la difusión y comunicación de la ciencia, la Revue d'Anthropologie des Connaissances y Trilogía Ciencia Tecnología Sociedad proponen este número temático con el fin de impulsar el debate, la consolidación y el fortalecimiento de la investigación por parte de la comunidad académica y científica de diversos países y disciplinas en torno al campo de la filosofía de la ciencia y la tecnología y su diálogo con los estudios CTS.

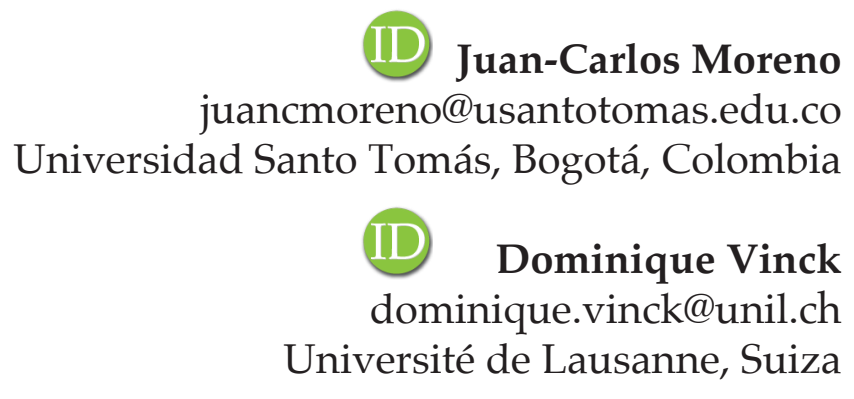

